\title{
Umsetzung integrativer Konzepte - eine Bestandsaufnahme
}

\author{
Markus Horneber ${ }^{\mathrm{a}} \quad$ Friedrich Overkamp ${ }^{\mathrm{b}} \quad$ Matthias Rostock $^{c}$ \\ ${ }^{a}$ Medizinische Klinik 5, Klinikum Nord, Nürnberg,

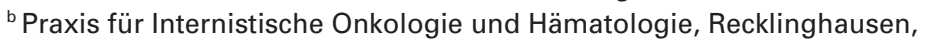 \\ ${ }^{c}$ Hubertus Wald Tumorzentrum, Universitäres Cancer Center, Hamburg, Deutschland
}

\section{Integrative Onkologie im Kontext eines universitären Cancer Centers \\ Verantwortlicher Autor: Matthias Rostock, Hamburg}

Komplementärmedizinische Verfahren werden in der Krebsbehandlung häufig eingesetzt. Genaue Angaben zur Anwendungshäufigkeit lassen sich einer Metaanalyse entnehmen, die im Rahmen eines systematischen Reviews durchgeführt wurde [1]. In die Analyse wurden 152 Studien aus 18 innerund außereuropäischen Ländern mit insgesamt mehr als 65000 Patienten einbezogen. Im Zeitraum 1979 bis 2009 haben demnach durchschnittlich $40 \%$ der Krebspatienten zusätzlich komplementärmedizinische Verfahren (CAM; complementary and alternative medicine) genutzt, was gemäß der Datenlage auch auf Deutschland zutraf. Die Autoren ermittelten ferner eine Zunahme der CAM-Nutzung von 25\% in den 1970er-Jahren auf durchschnittlich 49\% ab dem Jahr 2000. Patienten, die CAM anwenden, sind in der Mehrzahl weiblich, im jüngeren Lebensalter und haben einen höheren Sozialstatus [2, 3]. CAM-Anwender haben häufiger eine fortgeschrittene Krebserkrankung, eine ungünstigere Prognose sowie höhere aktuelle Schmerzwerte [3]. Sie leiden einer weiteren Studie zufolge eher unter Ängsten (besonders in Bezug auf Rezidivangst) und haben ein höheres Depressionsrisiko; eine grundsätzliche Verweigerungshaltung gegenüber der sogenannten Schulmedizin - obwohl häufig als Risiko angesehen - spielt aber generell keine wesentliche Rolle [4].

Patienten, die eine komplementärmedizinische Beratung am Universitären Cancer Center Hamburg (UCCH) wünschen, sind häufiger an Brustkrebs oder gynäkologischen Tumoren erkrankt, gefolgt von gastroenterologischen Krebserkrankungen (Abb. 1).

Das UCCH-Angebot «Naturheilkundliche und Komplementärmedizinische Beratung für Krebspatienten» besteht seit Anfang 2011; die Beratung erfolgt auf Zuweisung der Pa- tienten durch die Ambulanz- und Stationsärzte des UCCH bzw. den Kollegen anderer Abteilungen des Universitätsklinikums Hamburg-Eppendorf. Weitere Patienten kommen auf Empfehlung der mit dem UCCH kooperierenden onkologischen Praxen sowie örtlicher oder auch überregionaler Beratungsdienste oder wenden sich selbst an die Beratungssprechstunde.

\section{Die CAM-Beratung am Beispiel einer Patientin mit Brustkrebs}

Bei einer 39-jährigen Patientin wurde aufgrund eines invasiven Mammakarzinoms eine Teilresektion der Mamma vorgenommen und anschließend eine adjuvante Chemotherapie mit Fluoruracil, Epirubicin, Cyclophosphamid (FEC)/Paclitaxel eingeleitet. Die Patientin vertrug die Chemotherapie sehr schlecht und berichtete von anhaltender Übelkeit, Appetitlosigkeit und starkem Gewichtsverlust von insgesamt 8 kg Körpergewicht sowie Schleimhautentzündungen, Depression und Fatigue. Sie erwog deshalb, die Therapie nach 2 Therapiezyklen abzubrechen, sofern sich die Verträglichkeit nicht durch weitere, bisher nicht genutzte Maßnahmen - namentlich eine Misteltherapie - deutlich verbessern ließe. Während ihr behandelnder Onkologe dezidiert von einer begleitenden Misteltherapie abgeraten hatte, war ihr von einem telefonischen Beratungsdienst empfohlen worden, die Chemotherapie abzubrechen und eine rein komplementärmedizinische Nachbehandlung vornehmen zu lassen. In dieser belastenden Situation suchte sie unsere Beratungsstelle auf und bat um sachliche Information und Unterstützung.

Die Frage nach dem Nutzen der Mistel insbesondere bei Brustkrebs ist durchaus berechtigt. Gemäß Cochrane Review zur Misteltherapie, der auf einer Auswertung von 21 prospektiv-randomisierten Studien (davon 7 bei Mammakarzinom) beruht, gibt es Hinweise, dass die Misteltherapie sich überwiegend günstig auf bestimmte Aspekte der Lebensqualität

\begin{tabular}{|c|c|}
\hline KARGER & $\begin{array}{l}\text { (c) } 2012 \text { S. Karger GmbH, Freiburg } \\
0378-584 \mathrm{X} / 12 / 3517-0003 \$ 38.00 / 0\end{array}$ \\
\hline $\begin{array}{l}\text { Fax +49761 } 4520714 \\
\text { Information@Karger.de } \\
\text { www.karger.com }\end{array}$ & $\begin{array}{l}\text { Accessible online at: } \\
\text { www.karger.com/onk }\end{array}$ \\
\hline
\end{tabular}




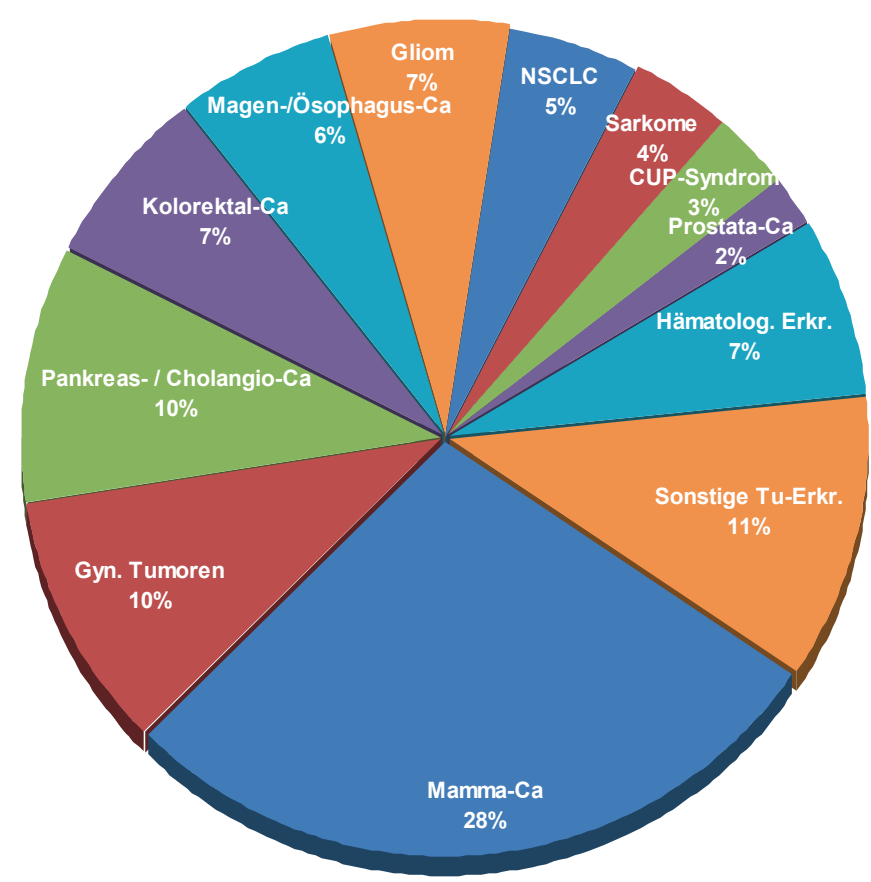

Abb. 1. Inanspruchnahme einer komplementärmedizinischen Beratung im UCCH Hamburg.

auswirkt [5]. 14 von 16 Studien mit Fokus auf Lebensqualität zeigten einen solchen Benefit. Eine Verlängerung der Lebenszeit bei Anwendung von Mistelpräparaten wurde lediglich in 6 von 13 Studien erreicht, wobei keine dieser Studien von höherer methodologischer Qualität war. In einer neueren, prospektiv-randomisierten Pilotstudie hatte sich erneut gezeigt, dass Patientinnen mit Mammakarzinom während einer adjuvanten Chemotherapie mit 6 Zyklen Fluoruracil, Doxorubicin, Cyclophosphamid (FAC) von der zusätzlichen Behandlung mit einem Mistelextrakt hinsichtlich ihrer Lebensqualität profitierten [6].

Nach Erläuterung der Datenlage entschied sich die oben vorgestellte Patientin für eine ergänzende Misteltherapie. Daraufhin konnte die Chemotherapie bei deutlicher Verbesserung der Verträglichkeit wie geplant fortgesetzt und komplett abgeschlossen werden.

\section{Granatapfel bei Prostatakarzinom, Weihrauchextrakt bei Hirntumor?}

Ein anderer Patient wandte sich mit der Frage an uns, ob komplementärmedizinische Maßnahmen nach radikaler chirurgischer Entfernung eines Prostatakarzinoms und bei langsam steigenden Prostata-spezifischen Antigen(PSA)-Werten, aktuell bei $0,19 \mathrm{ng} / \mathrm{ml}$ liegend, sinnvoll seien - insbesondere mit dem Ziel, eine Strahlentherapie zu umgehen bzw. zumindest hinauszögern zu können, da er große Angst vor möglichen Nebenwirkungen der Strahlentherapie hatte. $\mathrm{Zu}$ dieser Fragestellung war eine Phase-II-Studie in Kalifornien durchgeführt worden, die den Effekt von Granatapfelsaft untersucht hatte [7]. Die Studienteilnehmer hatten nach Primärthe- rapie eines Prostatakarzinoms erneut ansteigende PSA-Werte ( $>0,2$ und $<5,0 \mathrm{ng} / \mathrm{ml}$, median $2,23 \mathrm{ng} / \mathrm{ml}$ ) und nahmen täglich $240 \mathrm{ml}$ Granatapfelsaft zu sich. Zielparameter der Studie war die PSA-Verdoppelungszeit, die sich im Vergleich zum Studienbeginn von durchschnittlich 15,6 Monaten auf 54,7 Monate verlängerte $(\mathrm{p}<0,001)$. Bei etwa einem Drittel der 46 Studienteilnehmer sanken bei Anwendung des gut verträglichen Safts die PSA-Werte, die PSA-Reduktion betrug im Mittel $27 \%$. Granatapfelsaft enthält verschiedene Polyphenole, denen eine hohe antioxidative Wirkung zugeschrieben wird. Der Saft verfügt außerdem über schwache östrogene Effekte, besitzt entzündungs- und wachstumshemmende Eigenschaften und kann Apoptose induzieren.

Wir besprachen mit dem Patienten, dass leitliniengemäß eine Strahlentherapie mit sekundär kurativer Intention bei langsam ansteigendem PSA-Wert optimalerweise bei einem PSA-Wert zwischen 0,2 und $0,5 \mathrm{ng} / \mathrm{ml}$ eingeleitet werden sollte, in der bei ihm vorliegenden Situation ein Therapieversuch mit einem Granatapfelextrakt durchaus gerechtfertigt sei, dies aber unter engmaschiger PSA-Kontrolle vorzunehmen wäre. Leider führte die Einnahme eines Granatapfelextraktes bei diesem Patienten zu keiner Beeinflussung der PSA-Akzeleration - nach 6 Monaten war der PSA-Wert von 0,19 weiter auf $0,3 \mathrm{ng} / \mathrm{ml}$ angestiegen. Dennoch hatte er diese 6 Monate bei gleichzeitiger psychoonkologischer Betreuung so für sich nutzen können, dass er sich sehr viel angstfreier und entschiedener für die mittlerweile erfolgte Strahlentherapie entscheiden konnte.

Ebenfalls exemplarisch für die Aufgaben einer komplementärmedizinischen Beratungsstelle ist der Fall einer Patientin mit einem anaplastischen Astrozytom (Stadium WHO Grad III; WHO = World Health Organization). Primäres Symptom der Erkrankung waren epileptische Anfälle, die auch nach Teilresektion und nachfolgender Chemotherapie mit Temozolomid sowie antikonvulsiver Behandlung weiterhin auftraten. 6 Monate postoperativ begann die Patientin eigeninitiativ eine Behandlung mit Weihrauchextrakt (Boswellia serrata) in einer Dosierung von $3 \times 1600 \mathrm{mg} / \mathrm{Tag}$. Kurz darauf sistierten die einfach- bis komplex-fokalen Anfälle, was ihre behandelnden Ärzte primär auf die antikonvulsive Medikation zurückführten, sie selbst jedoch eindeutig durch die Weihrauchextrakt-Einnahme begründet sah. 3 Monate nach Beginn der Weihrauchextrakt-Therapie konnte in der kernspintomographischen Verlaufskontrolle unter fortlaufender Weihrauchextrakt-Einnahme auch eine Rückbildung des perifokalen Hirnödems dokumentiert werden.

Eine Fragebogenstudie des German Glioma Network an 6 neuro-onkologischen Zentren in Deutschland hat gezeigt, dass Patienten mit malignen Hirntumoren zu etwa 40\% auch auf komplementärmedizinische Verfahren setzen. Ein Viertel von ihnen wendet Weihrauchextrakte an, noch häufiger genutzt werden homöopathische Zubereitungen, Nahrungsergänzungsmittel sowie psychologische Interventionen (Abb. 2). 
Welche komplementären Methoden werden von Gliom-Patienten angewandt?

[Heese $\mathrm{O}$ et al. for the German Glioma Network: Complementary therapy use in patients with glioma. Neurology 2010;75:2229-2235]

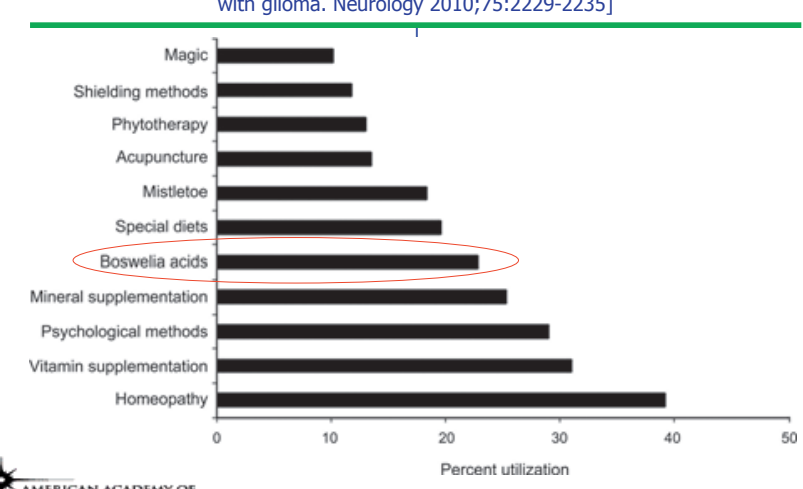

NMERCANACADEMY OF

Abb. 2. Komplementärmedizinische Verfahren bei Gliom-Patienten (nach [8]).

Weihrauchextrakt ist ein traditionelles Heilmittel in der Ayurvedischen Medizin und enthält als Hauptwirkstoffe Boswelliasäuren. Der Extrakt bewirkt eine Inhibition der 5-Lipoxygenase und der Topoisomerasen I und II sowie eine Induktion der Apoptose in verschiedenen Tumorzelllinien. Kürzlich wurden die Ergebnisse einer ersten randomisierten, placebokontrollierten Doppelblind-Studie zum Einsatz von Weihrauchextrakt bei Patienten mit Hirntumoren (Glioblastome oder Hirnmetastasen solider Tumoren) vorgestellt [9]. Die Verum-Gruppe erhielt den Weihrauchextrakt H15 in einer Tagesdosierung von 4200 mg $(3 \times 4$ Kapseln $)$. Primärer Studienendpunkt war das Volumen des zerebralen Ödems unter Strahlentherapie (30-60 Gy) bei Einnahme von Weihrauchextrakt bzw. Placebo. Bei Patienten aus der WeihrauchGruppe bildete sich das zerebrale Ödem signifikant stärker zurück und auch das Tumoransprechen unter Radiatio (gemessen an der größten Läsion) war signifikant besser. Die nach RECIST (Response Evaluation Criteria in Solid Tumors) beurteilte objektive Tumoransprechrate war allerdings in beiden Gruppen vergleichbar. Schwere Nebenwirkungen wurden bei Einnahme des Weihrauchextraktes nicht gesehen. Die Studienautoren ziehen eine positive Nutzen-RisikoBilanz für den Weihrauchextrakt, dessen Wirkung auf das zerebrale Ödem womöglich zu einer Einsparung von Steroiden führen könne. Weitere Untersuchungen zur Wirkweise des Extraktes sowie möglicher Antitumor-Effekte sollten in einer Phase-III-Studie untersucht werden, so die Autoren.

\section{Supportive Maßnahmen helfen bei Schluck- und Verdauungs-} beschwerden

Abschließend ein Beispiel, wie eine supportive komplementärmedizinische Behandlung einem Krebspatienten mit starken Schluckstörungen und chronischen gastrointestinalen Beschwerden helfen konnte. Auch wenn sich daraus keine allgemeine Empfehlung zum Vorgehen bei vergleichbarer Sympto-

matik ableiten lässt, so ließ sich doch in diesem Fall auf recht einfache Weise eine deutliche Beschwerdelinderung erreichen. Der hier zu beschreibende Patient hatte sich bei einem distalen Plattenepithelkarzinom des Ösophagus pT3 pN1 M1 (LK Truncus coeliacus) im Jahr 2005 nach Diagnosestellung einer abdominal-thorakalen Ösophagusresektion unterzogen und eine adjuvante Radiochemotherapie absolviert. Er litt fortan unter starken Schluckbeschwerden bei nahezu jeder Nahrungsaufnahme, krampfartigen Oberbauchbeschwerden und chronischer Diarrhö. Dies hatte einen Gewichtsverlust von $18 \mathrm{~kg}$ zur Folge und führte zu einer deutlichen Leistungseinschränkung mit dauerhafter Arbeitsunfähigkeit. Die mehrfache Bougierung einer Anastomosenstenose sowie eine Aryknorpel-Reposition hatten keine Besserung erbracht. Wir leiteten eine komplementäre Therapie mit Einnahme einer homöopathischen Zubereitung (Cuprum aceticum D4/Zincum valerianicum D4) und eines Probiotikums mit Laktobazillen und Bifidusbakterien ein und substituierten die Pankreasenzyme. Daraufhin besserten sich die seit über 6 Jahren konstant bestehenden Beschwerden rasch und anhaltend. Innerhalb von 12 Monaten konnte der Patient bei nur noch selten und in geringerer Ausprägung auftretenden Beschwerden wieder 6 $\mathrm{kg}$ an Körpergewicht zunehmen, was zu einer erheblichen $\mathrm{Zu}$ nahme von Leistungsfähigkeit und Lebensqualität geführt hat. Unter diesen Voraussetzungen plant der Patient, mittelfristig wieder seiner Berufstätigkeit nachgehen zu wollen.

\section{Komplementärmedizinische Beratung - welche Fragen sind zu stellen?}

Die genannten Beispiele sollen einen Einblick geben, wie weit das Spektrum der Patientenanliegen in der komplementärmedizinischen Beratung sein kann. Vor jeder Entscheidungsfindung in der Beratungssituation sind jedoch einige grundsätzliche Fragen zu klären, die sich in Kürze wie folgt formulieren lassen:

- Welche Therapien sind in der beim Patienten vorliegenden Situation gemäß dem aktuellen Stand des medizinischen Wissens zwingend notwendig?

- Was ist außerdem empfehlenswert? (z.B. Sport, Bewegung)

- Was könnte zusätzlich sinnvoll sein? (komplementäre Therapien je nach Fall)

- Was ist überflüssig?

- Was ist möglicherweise schädlich?

\section{Zum Stand der CAM}

Aus unserer bisherigen Beratungstätigkeit haben wir erfahren, dass aufseiten der Patienten ein großes Interesse an komplementärmedizinischen Verfahren besteht. Dies gilt auch für viele der zuweisenden Ärzte. Zugleich gibt es eine Reihe vielversprechender komplementärer Therapieansätze, die im Dialog mit dem Patienten kritisch gesichtet werden müssen. Bei der Beratung geht es darum, die Anliegen des Patienten zu erfassen und seine Fragen zur CAM möglichst umfassend anhand 


\section{Patientenbefragungen in onkologischen Schwerpunktpraxen}

"Wie beurteilen Sie die Gespräche mit dem Arzt?"

- Anteil "sehr gut" und "gut" in \% -

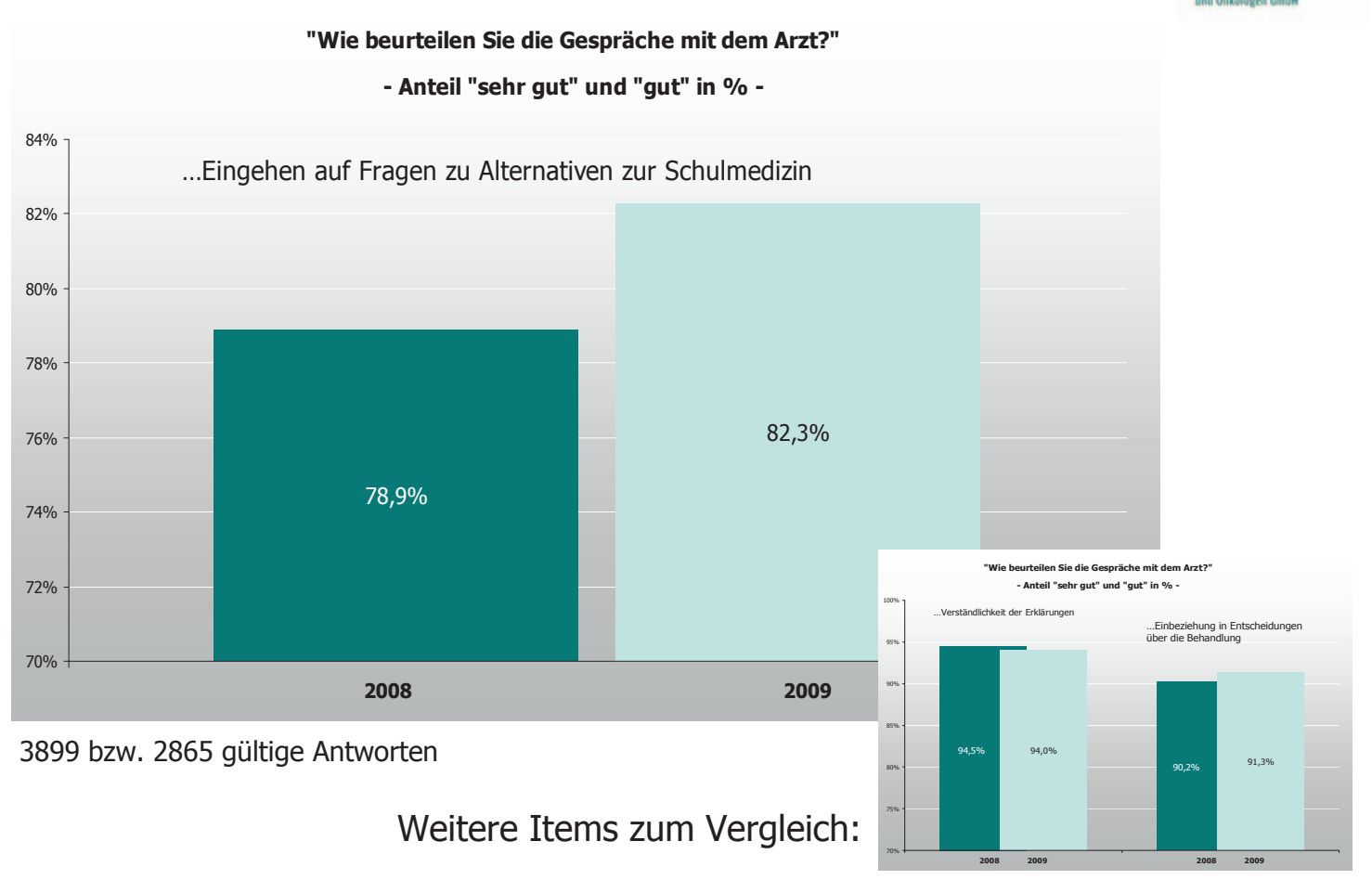

Abb. 3. WINHO-

Patientenbefragung zur Zufriedenheit mit den Gesprächen in onkologischen Schwerpunktpraxen der vorliegenden wissenschaftlichen Daten patientenzentriert und -verständlich zu beantworten. An mehreren, meist universitären Standorten wurden hierfür bereits Beratungsambulanzen eingerichtet. In den nächsten Jahren wird es unter Einhaltung eines hohen Qualitätsstandards um eine Netzwerkbildung für Versorgung, Forschung und Weiterbildung im Bereich der Komplementärmedizin in der Onkologie gehen mit dem Ziel, die langfristige Versorgung der Patienten zu verbessern.

\section{Integrative Onkologie im Kontext einer Praxis \\ Verantwortlicher Autor: Friedrich Overkamp, Recklinghausen}

Bis vor wenigen Jahren beschränkte sich das Angebot onkologisch ausgerichteter Praxen und Klinikambulanzen weitgehend auf die Schulmedizin. Komplementärmedizinische Verfahren wurden weder als Zusatzoption diskutiert noch wurden sie von den Patienten nachgefragt. Das hat sich grundlegend geändert. Sowohl vonseiten der Patienten wie auch vonseiten der behandelnden Ärzte besteht ein wachsendes Interesse an einer integrativen Krebstherapie, die mögliche neue Therapieansätze ohne bisheriges Schubladendenken (Schulmedizin versus Komplementärmedizin) auf den Prüfstand stellt. Dabei stellen sich 4 grundlegende Fragen:

- Welchen Stellenwert haben komplementäre Therapien im Kontext zur Schulmedizin in der onkologischen Praxis bzw. Klinikambulanz?
- Wo ist die Datenlage ausreichend und wo gibt es bereits eine Evidenz für ausgewählte Verfahren? Facharztpraxen vorhalten?

- Welche Methoden sollten nicht angeboten werden bzw. wovor sollten wir unsere Patienten schützen und warnen?

Das Wissenschaftliche Institut der Niedergelassenen Hämatologen und Onkologen (WINHO), das jährlich Qualitätsberichte über die Arbeit der niedergelassenen Onkologen herausgibt, führt unter anderem regelmäßig Patientenbefragungen durch, die auch Rückschlüsse auf den Stellenwert komplementärmedizinischer Verfahren im Praxisalltag erlauben. Gefragt nach der allgemeinen Gesprächsqualität zwischen Arzt und Patient haben mehr als 90\% der Befragten wiederholt angegeben, die Gespräche seien im Hinblick auf die Verständlichkeit der Erklärungen gut bis sehr gut gewesen. Jeweils mehr als $90 \%$ der Patienten fanden außerdem, dass sie in Therapieentscheidungen «gut» bis «sehr gut» einbezogen worden seien. Der Grad an Zustimmung zu diesen Punkten ist seit Jahren konstant. Anders verhält es sich mit Fragen der Komplementärmedizin (Abb. 3). Hier haben die Praxen in Sachen Information innerhalb kurzer Zeit aufgeholt: Fragen nach Alternativen zur Schulmedizin wurden im Jahr 2009 zu über $80 \%$ «gut» oder «sehr gut» beantwortet. Ob sich diese menden Umfragen unter unseren Patienten zeigen. Mit dem WINHO kooperieren derzeit 394 Fachärzte aus 209 onkologi-
- Welche komplementärmedizinischen Angebote sollten erfreuliche Entwicklung weiter fortsetzt, werden die kom- 


\section{Patientenbefragungen in onkologischen Schwerpunktpraxen}

Abb. 4. WINHO

Patientenbefragung zur Thematisierung von Ernährung,

Bewegung und sozialen Faktoren.

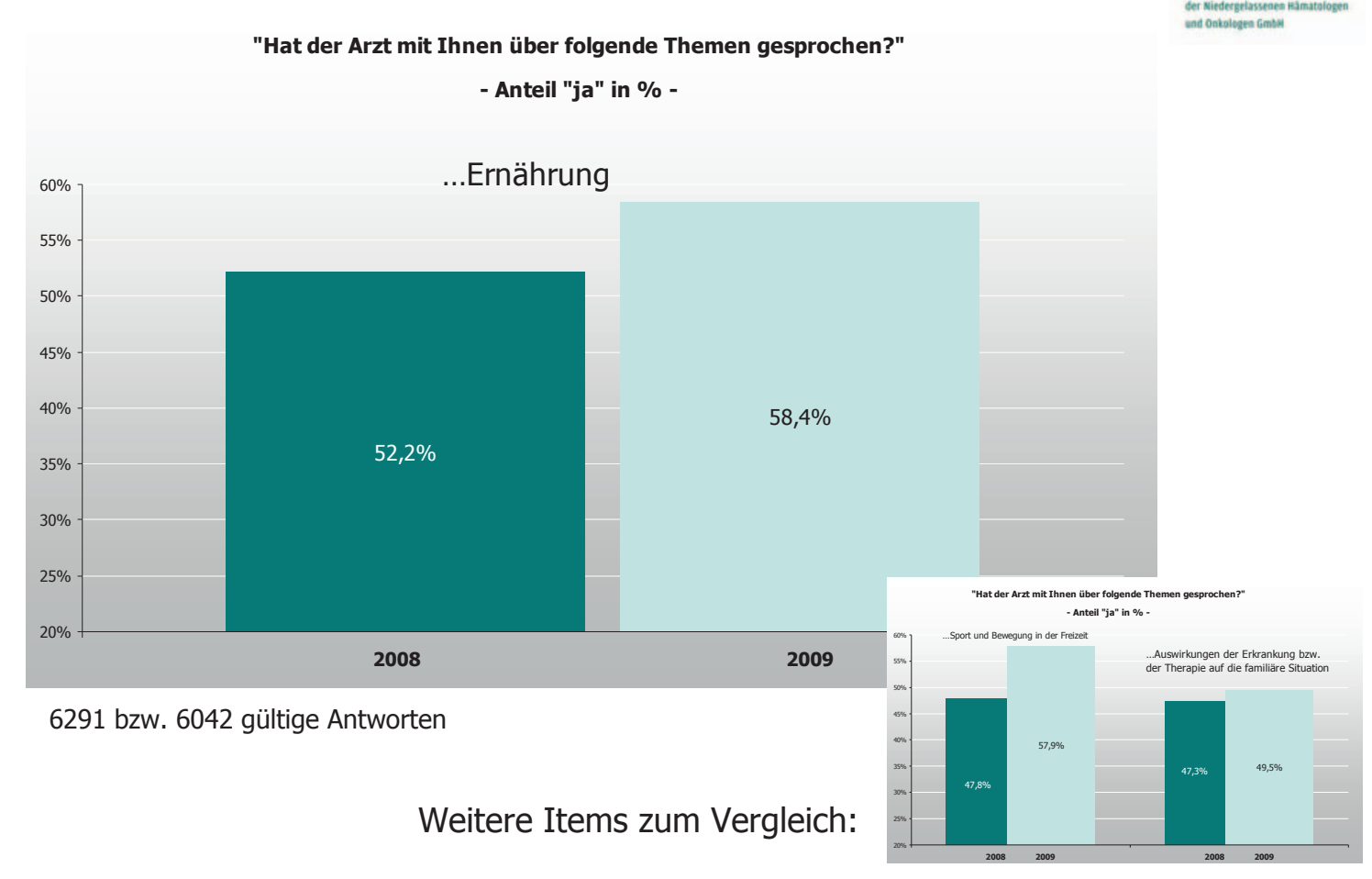

schen Schwerpunktpraxen. Die Patientenbefragung dürfte somit für die deutsche Praxislandschaft repräsentativ sein.

Auch bei Fragen zu Ernährung und Sport/Bewegung zeigt sich der Trend einer zunehmenden Thematisierung (Abb. 4). Mittlerweile wird in vielen Praxen eine spezielle Ernährungsberatung angeboten, in der auf die individuelle Situation im Zusammenhang mit Krebserkrankung und -therapie eingegangen wird. Auch der Bereich «Sport und Bewegung» spielt eine erkennbar zunehmende Rolle bei der Erörterung zusätzlicher Möglichkeiten zur Bewältigung von Erkrankung und Nebenwirkungen. Die sozialen Aspekte einer Krebserkrankung werden im Zusammenspiel mit Selbsthilfegruppen und durch das wachsende Angebot von Seminaren für Patienten und Angehörige thematisiert.

Viele Hämatologen und Onkologen kooperieren mit Selbsthilfeeinrichtungen. Oftmals werden Informationsangebote gemeinsam mit Betroffenen aus Selbsthilfegruppen gestaltet. Laut Umfrage in 155 Praxen werden von einem Drittel auch eigene Patientenseminare angeboten (Abb. 5). Bei diesen Veranstaltungen geht es nicht nur um «schulmedizinische» Standardtherapien, sondern um alle Facetten einer Krebserkrankung einschließlich komplementärmedizinischer Therapie- und Bewältigungsstrategien.

Auch werden von mehr als einem Drittel der dem WINHO angeschlossenen Praxen den Patienten regelmäßig weitere Zusatzangebote gemacht, insbesondere im Bereich der schon erwähnten Ernährungs- bzw. Diätberatung und der psychoso- zialen Beratung (Abb. 6). Zweifellos könnte das Angebot noch ausgeweitet werden, besonders was die sportliche Aktivität und Psychoonkologie betrifft. Es handelt sich bei all diesen Angeboten jedoch um reine Zusatzleistungen, deren Kosten weder von den Kassen erstattet noch den Patienten in Rechnung gestellt werden.

Welche Angebote die einzelnen Praxen vorhalten, hängt letztlich vom jeweiligen Schwerpunkt der Einrichtung ab. Eine Praxis, die sich z.B. auf die Betreuung von Frauen mit Brustkrebs spezialisiert hat, wird sich eher mit Yoga und Akupunktur als zusätzlichen Optionen befassen, zumal beides auch von der Arbeitsgemeinschaft Gynäkologische Onkologie (AGO) zur Linderung von Beschwerden unter endokriner Therapie empfohlen wird [10]. Insofern sind diese Formen der Komplementärmedizin evidenzbasiert, da die Empfehlung der AGO auf der Grundlage kontrollierter Studien zum Nutzen von Yoga und Akupunktur ausgesprochen wurde. Viele unserer Patienten wenden zusätzlich zu den ihnen verordneten Therapien auch Phytotherapeutika an, entweder nur bei akuten Beschwerden oder auch regelmäßig. Da viele Krebserkrankungen chronisch verlaufen, ist die Langzeitanwendung von additiven (pflanzlichen) Arzneien ein neuer Aspekt, der in der Therapieführung und -kontrolle beachtet werden muss. Allerdings wird die Nutzung von Phytotherapeutika in der onkologischen Praxis von den Patienten selten angesprochen, da sie die Präparate andernorts erhalten und naturheilkundlich ausgerichtete Ärzte zurate ziehen. Hier 
Abb. 5. Aus dem Qualitätsbericht 2010 des WINHO: Förderung von Selbsthilfe und Patientenberatung [11].

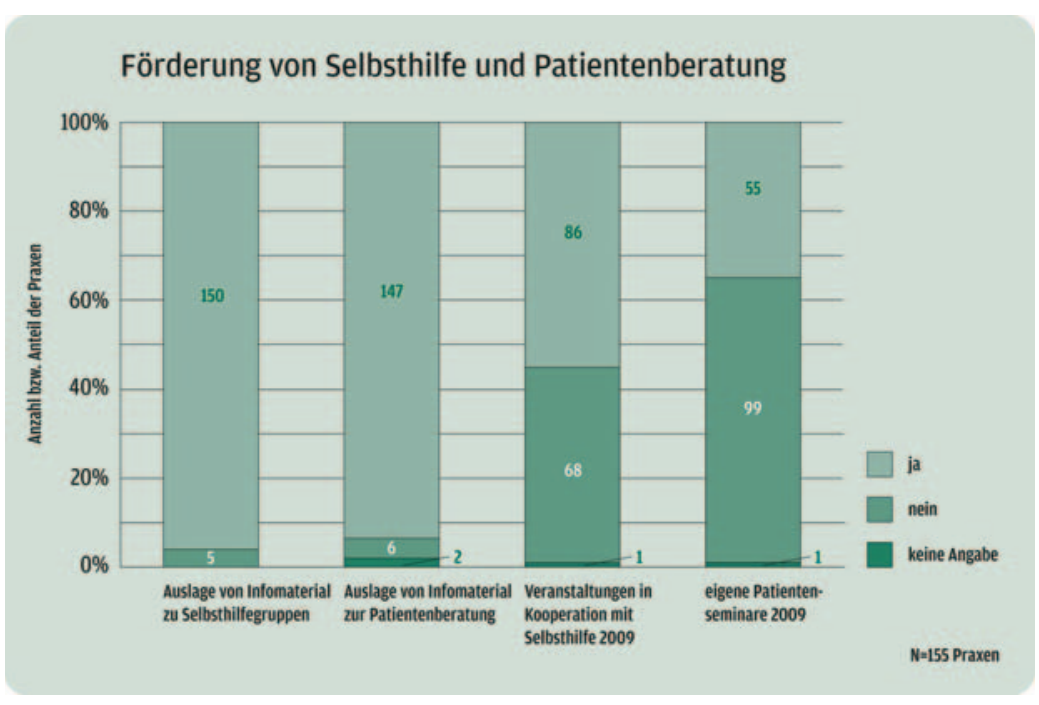

Qualitätsbericht 2010 der onkologischen Schwerpunktpraxen

Abb. 6. Fortsetzung Qualitätsbericht 2010.

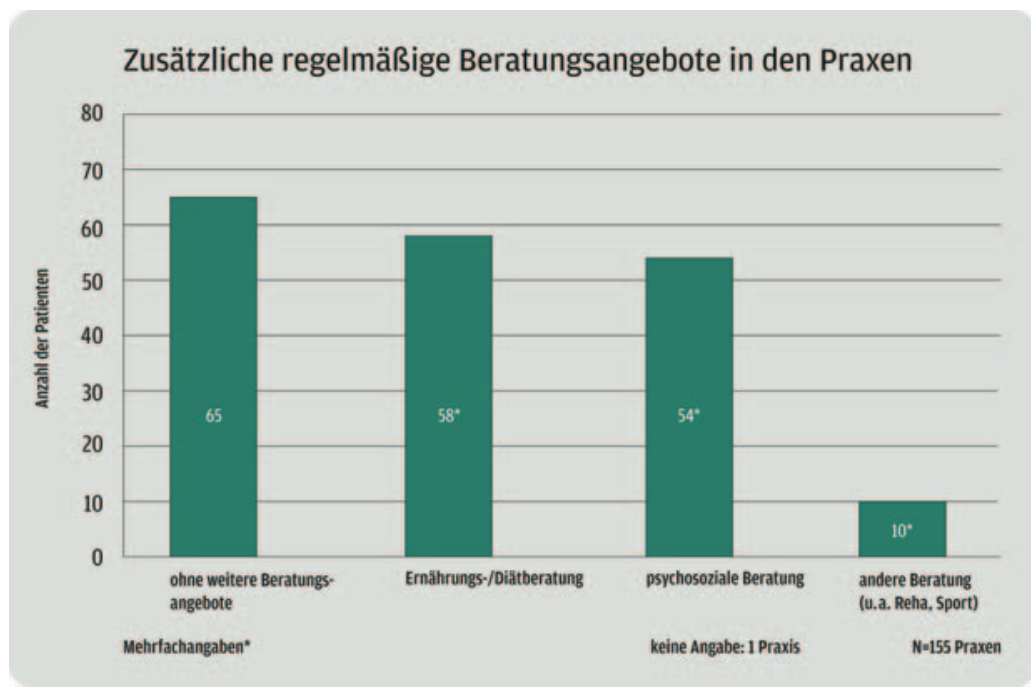

wäre eine engere, auch fachübergreifende Zusammenarbeit wünschenswert, insbesondere um bei möglichen Arzneimittelinteraktionen frühzeitig gegensteuern zu können. An verschiedenen Orten haben sich bereits Netzwerke gebildet, die auf die ganzheitliche Betreuung von Patienten abzielen.

\section{Komplementärmedizin kritisch umsetzen}

Zwar sind viele Patienten an komplementärmedizinischen Verfahren interessiert, sie erwarten von ihrem behandelnden Onkologen aber nicht, dass er auch auf diesem Gebiet ver- siert ist. Ein Druck, bestimmte Zusatzangebote vorzuhalten, besteht unseres Erachtens nicht. Zunehmend wichtiger wird jedoch die Bereitschaft, bei Bedarf komplementärmedizinische Zusatzangebote zu vermitteln.

Es sei dahingestellt, welchen Platz komplementäre Verfahren in Therapie und Beratung künftig beanspruchen sollten. Vordringliche Aufgabe der Onkologen bleibt die Behandlung der Patienten nach dem aktuellen Wissensstand. Eine Komplementärmedizin, die konsensfähige evidenzbasierte Kriterien erfüllt, wird auch Eingang in die Praxen finden. 
Abb. 7. Beweggründe von Krebspatienten zur Nutzung von Komplementärmedizin. Untersuchung von Umfragen aus europäischen Ländern von 1979 bis 2009 (unveröffentlichte Daten aus [1])
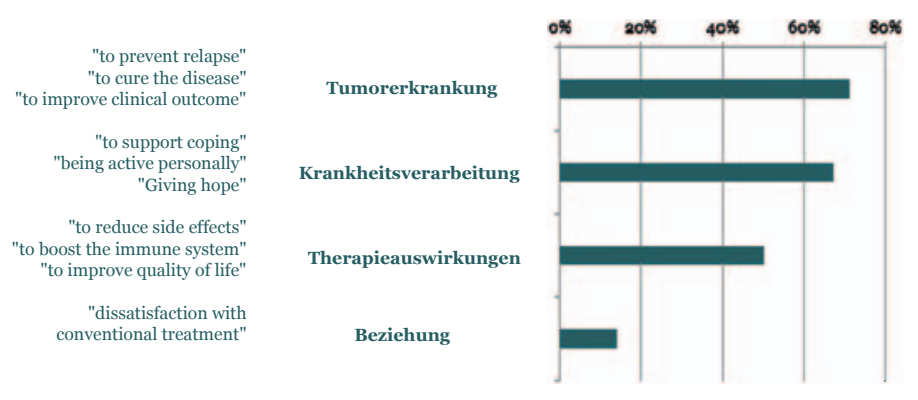

\section{Die Bedürfnisse der Patienten \\ Verantwortlicher Autor: Markus Horneber, Nürnberg}

Aus psychologischer Sicht sind Bedürfnisse der «Motor unseres Handelns», die «Beweggründe, die alle anderen Funktionen wie Wahrnehmung, Denken und Handeln in Gang zu setzen vermögen» [12]. Unser aller Bestreben ist es, grundlegende Bedürfnisse wie solche nach Orientierung und Kontrolle, Wohlbefinden, sozialer Bindung und Stärkung unseres Selbstwerts zu befriedigen. Darauf sind unsere Handlungen ausgerichtet und gelingt uns dies, sind wir zufrieden und glücklich; bleiben diese Bedürfnisse jedoch unerfüllt, sind wir unzufrieden, rastlos und angespannt. Bedürfnisse sind somit als zentrales Element der Lebensqualität des Menschen zu betrachten [13]. Gleiches gilt für Patienten mit Krebserkrankungen. Nicht erfüllte oder als nicht erfüllt empfundene Bedürfnisse, insbesondere solcher nach Information und Mitbestimmung, nach guter Beziehung mit den behandelnden Ärzten und nach sozioemotionaler Unterstützung, erzeugen einen Spannungszustand, der zumindest die Arzt-PatientenBeziehung, womöglich aber auch die ganze Behandlung belasten kann [14]. Daher darf der Erfolg ärztlicher Behandlung und Betreuung nicht allein an objektiven Parametern wie Überlebenszeiten oder Ansprechraten gemessen werden. Vielmehr gilt es, die individuelle, subjektive und emotionale Seite der Betroffenen gleichermaßen zu berücksichtigen und dem Einzelnen die Möglichkeit zu geben, die für ihn zentralen Themen einzubringen und sie nach persönlicher Wichtigkeit zu ordnen.

In einer aussagekräftigen, deutschlandweiten Umfrage wurden über 700 Patientinnen mit Brustkrebs zu ihren Bedürfnissen und Erwartungen in Bezug auf die Behandlung und die Patienten-Arzt-Beziehung befragt. Auf die Frage, welche Änderungen sie für am wichtigsten hielten und vornehmen würden, wenn sie die Brustkrebsbehandlung verbessern sollten, wurde der Wunsch nach einer stärkeren Integration der Komplementärmedizin in die Behandlung am häufigsten geäußert («Doctors should offer complementary medicine as well»), gefolgt von dem Bedürfnis nach mehr Zeit für Gespräch und Austausch («Doctors should take more time explaining things») und dem Anliegen nach guter Zusammenarbeit zwischen den Fachdisziplinen («Better cooperation among doctors») [15]. Diese Ergebnisse weisen nicht nur auf den hohen Wert hin, den Patienten auf eine vertrauensvolle Beziehung zu den Ärzten legen, sondern verdeutlichen auch das starke Bedürfnis nach individuellen Heilungskonzepten, das mit dem oben genannten Wunsch nach einer Integration der Komplementärmedizin ausgedrückt wird.

Entsprechend diesem Wunsch, werden Verfahren der Komplementärmedizin bereits von vielen Krebspatienten genutzt, wie eine systematische Übersichtsarbeit berichtet [1]. In einer bisher unveröffentlichten Arbeit wurden die Beweggründe für die Nutzung der Komplementärmedizin in 74 Umfragen ausgewertet, die in diese Übersichtsarbeit eingeschlossenen waren. Hier zeigte sich, dass an die Komplementärmedizin durchaus keine anderen Erwartungen gestellt werden als an konventionelle onkologische Behandlungen (Abb. 7).

Eine qualitative Untersuchung der Beratungsgespräche zur Komplementärmedizin in der Onkologie, die seit vielen Jahren am Klinikum Nürnberg mit der Unterstützung der Deutschen Krebshilfe angeboten werden, fand folgende Hauptbeweggründe, weshalb Patienten ärztlichen Rat zu komplementärmedizinischen Behandlungen suchten [16]:

- Minderung von Symptomen und Belastungen,

- Schutz vor Nebenwirkungen der konventionellen onkologischen Therapien, 


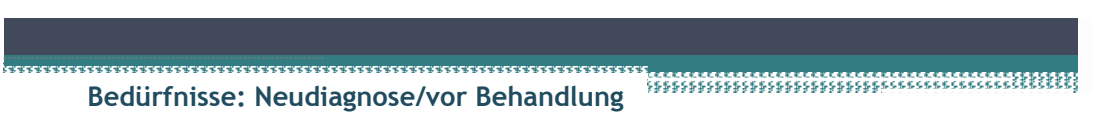

Bedürfnisse: Neudiagnose/vor Behandlung

Abb. 8. Kategorien von Beweggründen, weshalb eine komplementärmedizinische Behandlung unmittelbar nach der Erstdiagnose und vor der onkologischen Behandlung gesucht wurde.

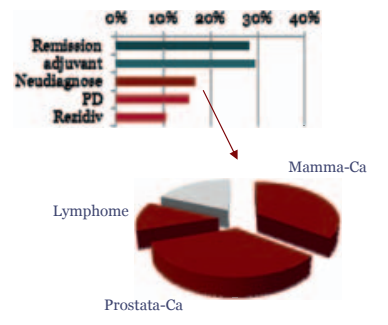

... nach Behandlungsalternativen

"ist die Galvanotherapie eine Alternative zur Operation?"

... nach Klärung von Widersprüchen

"ich bin sehr verunsichert, Hausärztin, Onkologe, Heilpraktiker, jeder sagt etwas anderes"

... nach Schutz vor Nebenwirkungen

"Was kann tun, dass ich die OP gut überstehe?"

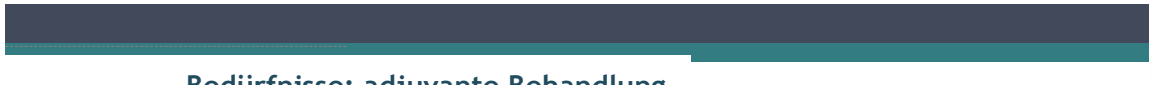

Bedürfnisse: adjuvante Behandlung

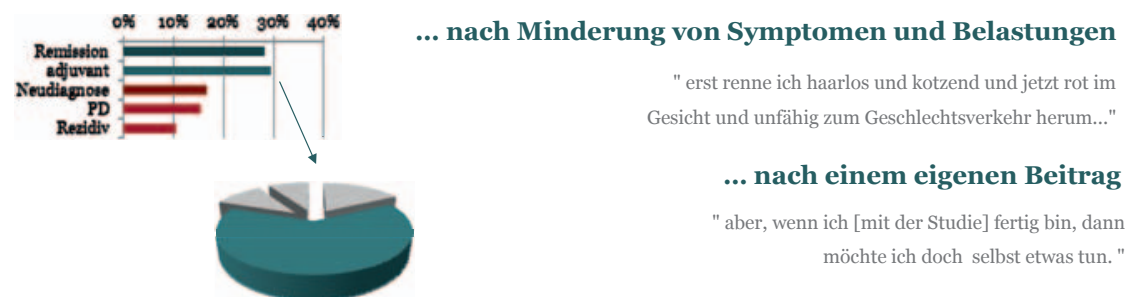

Mamma-Ca

... nach Hilfe bei Informationsvielfalt

"...habe natürlich viel gelesen, aber ich möchte mich nicht verzetteln und brauche Ihren Rat"

... nach Behandlungsalternative

Abb. 9. Kategorien von Beweggründen, weshalb eine komplementärmedizinische Behandlung während der adjuvanten Chemotherapie bei Brustkrebs gesucht wurde.
"Kann ich mich statt dieser Chemo auch impfen lassen gegen meinen Tumor?"
- Suche nach Behandlungsalternativen zu konventionellen onkologischen Therapien,

- Klärung von Widersprüchen bzw. Hilfe bei Informationsvielfalt,

- Wunsch nach einem eigenen Beitrag zur Gesundheitsförderung.

Darüber hinaus zeigte die Untersuchung, dass sich die Beweggründe für das Suchen nach Komplementärmedizin je nach Krankheitssituation deutlich unterschieden:

War die Krisensituation der Neudiagnose und das Anstehen der ersten Behandlungsentscheidung der Anlass für das Gespräch, standen Fragen nach Alternativen zur konventionellen Therapie aus dem Bereich der Komplementärmedizin stark im Vordergrund (Abb. 8).

Würde in diesen Situationen von Ärzten nicht wahrgenommen, dass die Frage nach «der Alternative» nicht zuerst Zwei- fel an der Wirksamkeit der empfohlenen konventionellen Therapie ausdrückt, sondern vielmehr dem Bedürfnis entstammt, Orientierung und Kontrolle in einer existenziell erschütterten Situation zurückzugewinnen, drohte Enttäuschung auf beiden Seiten, bis hin zum Abbruch der Beziehung und unter Umständen auch der anstehenden Behandlung [17].

Waren die Patienten allerdings in einer weniger krisenhaften Situation, so z.B. während einer adjuvanten Chemotherapie, standen Fragen zum Umgang mit Nebenwirkungen der Therapie und nach Möglichkeiten, einen eigenen Beitrag zur Gesundheitsförderung zu leisten, im Vordergrund, was als Ausdruck für andere grundlegende Bedürfnisse gesehen werden kann, nämlich solchen nach Wohlbefinden und Stärkung des Selbstwerts (Abb. 9).

Zusammengefasst kann ein Umgang mit der Komplementärmedizin in der Onkologie, der die zugrunde liegenden Be- 
dürfnisse und Beweggründe der Patienten im Sinne einer Integrativen Onkologie einbezieht, in besonderer und unmittelbarer Form die Anpassungsvorgänge der Betroffenen im Rahmen der Krankheitsauseinandersetzung und des Gesundungsprozesses deutlich werden lassen und den Betroffenen helfen, sich auf die je nach Situation unterschiedlichen Bedeutungen für die Patienten-Arzt-Beziehung einzustellen.

\section{Disclosure Statement}

Es bestehen keine Interessenkonflikte.

\section{Literatur}

1 Horneber M, et al.: How many cancer patients use complementary and alternative medicine: a systematic review and metaanalysis. Integr Cancer Ther 2011; DOI: $10.1177 / 1534735411423920$.

2 Paltiel O, et al.: Determinants of the use of complementary therapies by patients with cancer. Clin Oncol 2001;19:2439-2448.

$\checkmark 3$ Nagel G, et al.: Use of complementary and alternative medicine by patients with breast cancer: observations from a health-care survey. Support Care Cancer 2004;12:789-796.

4 Burstein H, et al.: Use of alternative medicine by women with early-stage breast cancer. N Engl J Med 1999;340:1733-1739.

5 Horneber MA, et al.: Mistletoe therapy in oncology. Cochrane Database Syst Rev 2008;(2):CD003297.

6 Tröger W, et al.: Quality of life and neutropenia in patients with early stage breast cancer: a randomized pilot study comparing additional treatment with mistletoe extract to chemotherapy alone. Breast Cancer (Auckl) 2009;3:35-45.

7 Pantuck AJ, et al.: Phase II study of pomegranate juice for men with rising prostate-specific antigen following surgery or radiation for prostate cancer. Clin Cancer Res 2006;12:4018-4026.

8 Heese $\mathrm{O}$, et al.: Complementary therapy use in patients with glioma: an observational study. Neurology 2010;75:2229-2235.

9 Kirste S, et al.: Boswellia serrata acts on cerebral edema in patients irradiated for brain tumors: a prospective, randomized, placebo-controlled, double-blind pilot trial. Cancer 2011;117:3788-3795.

10 AGO-Leitlinie Mammakarzinome: Komplementäre Therapie im Kontext von Integrativer Onkologie. Hormontherapie nach Mammakarzinom und Alternativen «Survivorship». www.ago-online.de/fileadmin/downloads/leitlinien/ mamma/g_mamma_11_1_0_d_25_complementary_therapy.pdf.

11 WINHO Qualitätsbericht 2010. www.winho.de/fileadmin/Downloads/QS Bericht_2010.PDF.

12 Asanger R, Wenninger G: Handwörterbuch Psychologie. Weinheim, Verlag Beltz PVU, 1999.

13 Stucki C, Grawe K: Bedürfnis- und motivorientierte Beziehungsgestaltung. Psychotherapeut 2007;52:16-23.

14 Fischbeck S: Bedürfnisse der Brustkrebs-Patientin. Wie mein Arzt mir helfen könnte. Würzburg, Verlag Königshausen und Neumann, 2002.

15 Oskay-Oezcelik G, et al.: Breast cancer patients' expectations in respect of the physician-patient relationship and treatment management. Results of a survey of 617 patients. Ann Oncol 2007;18:479-484.

16 Horneber M, et al.: Telephone consultation for complementary and alternative medicine in cancer: who calls and what do they need? Patient Educ Couns 2012; submitted.

17 Goldberg RJ: Systematic understanding of cancer patients who refuse treatment. Psychother Psychosom 1983;39:180-189. 